\title{
2. Key Levels of Biocommunication
}

\author{
Guenther Witzany \\ Telos-Philosophische Praxis, Vogelsangstrasse 18c \\ 5111-Buermoos, Austria \\ witzany@sbg.at
}

Organisms actively compete for environmental resources. They assess their surroundings, estimate how much energy they need for particular goals, and then realize the optimum variant. They take measures to control certain environmental resources. They perceive themselves and can distinguish between "self" and "non-self." Current empirical data on all domains of life indicate that unicellular organisms such as bacteria, archaea, giant viruses, and protozoa as well as multicellular organisms such as animals, fungi, and plants coordinate and organize their essential life functions through 등 signaling processes. Signaling allows for real life coordination and o organization and is a communicative action in which speciesspecific behavioral patterns and sign repertoires are used. Cells, tissues, organs, and organisms that communicate share several key levels that are essential to all life forms and which serve as a uniform tool for investigating biocommunication. Complementary to this, active biocommunication depends on the deoxyribonucleic acid (DNA) storage medium and the agents that generate coherent content of nucleic acid sequences. Therefore natural genome 
editing is not the result of replication errors but of group interactions of competent ribonucleic acid (RNA) agents.

\section{Introduction}

For centuries communication has been limited to humans, whereas non-human living organisms have been assumed to function mechanistically as machine-like stimulus reaction automatons determined by genetic programs. This mechanistic perspective has also dominated the description and explanation of human behaviorism within various sender receiver narratives: first a sender has a thought, and then it is coded into signs/codes and transmitted via a channel to a receiver who decodes it in accordance with his/her private value system. Similar explanatory models have dominated the mathematical theory of language, systems theory, biolinguistics, bioinformatics, and biosemiotics (Popov et al., 1996; Nowak and Krakauer 1999; Nowak et al., 2000; Nowak et al., 2001, 2002; Eigen and Winkler 1983, Eigen, 2013; Searls, 2002; Chomsky, 1964; Ji, 1999; Barbieri, 2001). The focus of these concepts varies from physical/chemical properties of signs and transfer channels (biolinguistics, biosemiotics), to codes (biology), to systems of higher and lower orders, to material reality depicted as the ideal in mathematical formalizations (bioinformatics). Because organisms, especially animals with central nervous systems and brain organs, are built of chemical molecules that follow physical laws, the best reality depicting language is mathematics, in which brain architecture and its function are represented (Shannon and Weaver 1949; Eigen and Winkler, 1983). Theories that propose other entities than atoms and molecules as essential tools of life are guilty of "vitalism"-like narratives (Venter, 2013). Therefore, we must investigate all systems in which a universal syntax - that represents the logical order of material reality - can be found at the root of all languages. Logically, computation methods are the best tools for research, because organisms can be said to depict hard drives and DNA the software (Venter, 2013).

This $20^{\text {th }}$ century narrative is outdated, however (Witzany, 2012a, 2012b; Witzany and Baluška, 2012a, 2012b; Baluška and Witzany, 2013). Empirical data indicate communication is primarily a social 
interaction using languages/codes, i.e., social interactions between living agents that share a real life world's traditions and environmental circumstances (Tomasello, 2008). Experience and the organization, and coordination of everyday real life practice determine communication patterns (Habermas, 1984, 1987, 1994). Language serves as a tool for exchanging relevant contents, i.e., meanings. Most importantly, however, the social interaction of communication is a real action - intended to convince, to persuade, to obey, to share social experience, feelings and thoughts, reciprocal or imperative (Austin, 1975; Searle, 1976). Neurobiological research of the last decades demonstrated that the social brain organs of children learn through observations and mimicry, and consequent feedback from adult group members (Tomasello, 2008). The early experience of coherent complementarity between cognition and emotion in real life situations is essential to these basic experiences and cannot be predicted or computed by mathematical algorithms. During socialization, children not only learn how to use a small vocabulary to express their feelings but also build sentences to coordinate with speech acts. Through observations and mimicry they learn to combine words with correct meanings; they do things with words. Later in their development, group members who share communicative and linguistic competence describe observations through constative speech acts or communities install laws that orientate common interactions and goals as regulatory speech acts. In everyday life, we do things with words that have serious consequences.

Utterances represent an obvious superficial grammar, but what we mean or what we intend depends on various circumstances, intentions, and goals. We express our meaning through gestures, eye movements, and vocal focusing (emphasis). Therefore, we have a limited number of signs for creating unlimited number of sentences. Additionally we may vary the meaning of identical sentences, which is not represented by syntax (see Fig. 1). This means that communication as a social interaction differs absolutely from the solipsistic sender-receiver narrative as well as the information-transfer narrative (Wittgenstein, 1953; Austin, 1975; Searle, 1976; Habermas, 1994).

Sender-receiver narratives confuse their language of description with reality or how to interconnect thoughts, language, and 


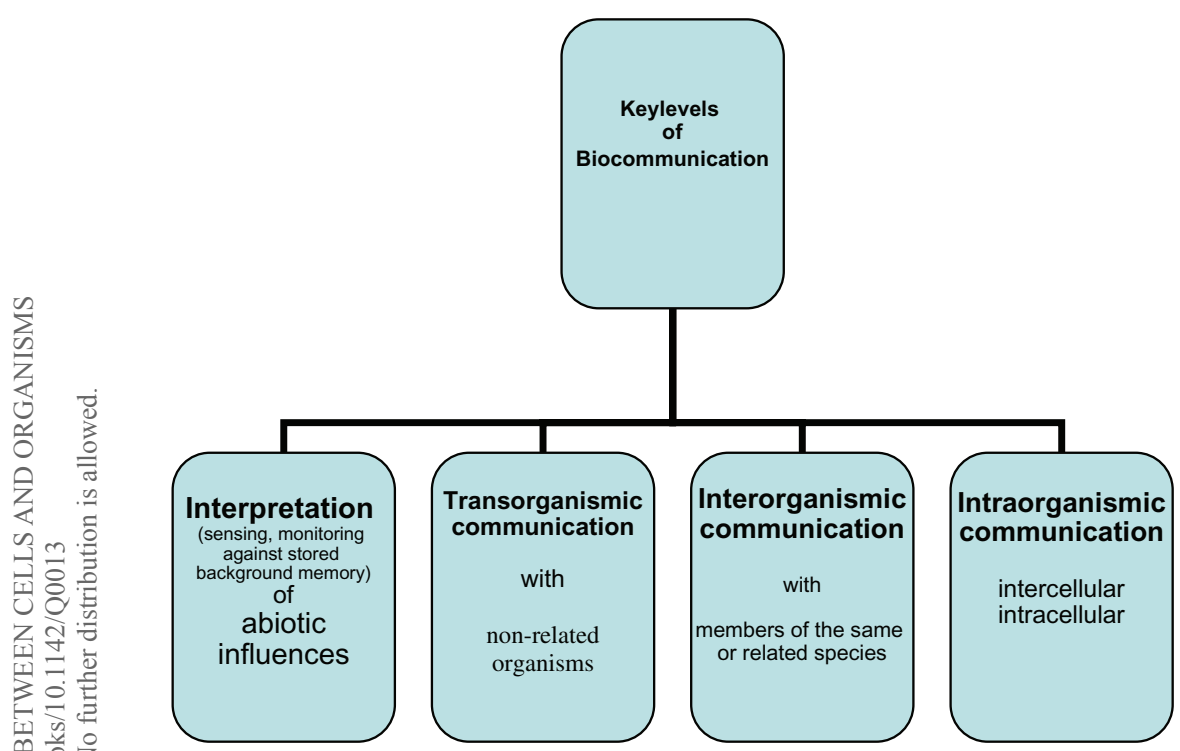

Figure 1. The key levels of biocommunication.

observations. This is the real problem with all metaphysical approaches such as objectivism, realism, and even ontology: not being aware, that the basic needs they describe do not depict physical objects (McCarthy, 1984). Yet sentences of languages solely represent the language of description, its background paradigm, and, in the case of sciences, the current consensus of discipline agreements (Kuhn, 1967). At the basis of all reasoning, a good basis for knowledge about the world, must be a coherent model of language and communication. Scientific knowledge is the result of exchange of arguments within scientific communities. I have outlined in previous works the reasons why none of the above-mentioned concepts can explain the evolution and function of languages used in communicative interactions (Witzany, 1995, $2000,2010)$. The core reasons are that these concepts cannot sufficiently explain:

- Simultaneous understanding of identical meanings in two interacting partners, as expressed in successfully coordinated activity; 
- Differentiation between deep and superficial grammar of a statement along with differentiation between locutionary, illocutionary, and perlocutionary speech acts;

- The de novo generation of coherent and context-dependent sentences.

\section{Roots of Scientific Knowledge}

In contrast, this article will try to start at the bottom. What can we be sure to know as scientific knowledge? How do we assemble empirical data and how do we construct experimental setups free from personal values? What is it that we define as empirical significance? Is there any unequivocal definition of empirical significance? What are sure definitions of observation, description, understanding, theory? What definitions of the objective world, subjective world, cognitive perception, and dispositions are unequivocal? Is there any reality that cannot be described? What can we be unequivocally sure we all agree with founded and justified arguments? What are the tools that we can rely on in discussion of these problems?

These are some of the core assumptions and questions that were questioned in the early $20^{\text {th }}$ century when scientists tried to construct an unequivocal scientific language clearly delimited from non-scientific languages such as theology, poetry, and metaphysics. The discussion lasted until the late 1980s and produced definitive results (Witzany, 2000, 2010).

- The only knowledge we can be undoubtedly sure is, that if we want to discuss or speak about something in the world we need a commonly shared sign system (language).

- No knowledge, no communication, no exchange of any information, no idea, or thought can be generated without a commonly shared language. Even if one would doubt in this, how could he present his critics? We do not have ideas/thoughts that we afterwards transfer into sentences as they are inherent linguistically built and constructed.

- The only sure knowledge is how language and communication function. They are both subjects and objects of our language-based 
utterances, so we can clearly identify the prerequisites of communication and language, and what is missing if language and communication do not function.

- One person alone cannot invent a language. Languages emerge from groups of living agents who need tools to coordinate behavior and organize real life needs. Languages are used in communicative acts. Because a private language is impossible in principle, communication is inherently a kind of social interaction. Private thoughts follow social interaction in which we learn communicative and linguistic competences and do not predate them.

We term communicative competence as the ability to correctly initiate social interactions. We term linguistic competence as the ability to correctly generate sentences.

Every natural language/code needs groups of living agents who use this language in real life world to coordinate and organize. This means in natural languages there cannot be such things as context-free grammar. This is an artificial construction. Prior to all artificial languages such as languages of scientific disciplines, humans learned via socialization processes in everyday language how to combine actions with words and correct meanings, the complementary interaction of cognitive and emotional developments. Private thought, as well as artificial scientific languages, follows as a second step and does not predate social experiences of communicative sign-mediated interactions.

\section{3. "If a Lion Could Speak, We Could Not Understand Him"}

It is true that if lions could speak we would be unable to understand their conversation (Wittgenstein, 1953). The reason lies within the essential features of languages. They emerged out of functional behaviors interconnected with social interactions. As humans we must be lions, which is clearly impossible, to grow up within lion families, share their history. To understand a speciesspecific utterance someone must be a specific part of the 
community to understand what is meant by the signal in detail. This was Wittgenstein's understanding.

Yet there is another approach that helps us to understand signaling processes in non-human living beings. Charles Sanders Peirce suggested that to identify the meaning of signs, words, and language-like structures, "we have, therefore, simply to determine what habits it produces, for what a thing means is simply what habits it involves" (Peirce, 1923). This is a similar definition to Wittgenstein's "The meaning of a word is its use" (Wittgenstein, 1953) the core assumption of pragmatics that the function is required to identify the meaning, as outlined by the best interpreter of Ludwig Wittgenstein, John Austin, in How to Do Things With Words (1975). In biocommunication research, this means we search for species-specific life worlds with genera, families, and species:

- Observations on how they live (ecology, niche construction).

- Finding excreted molecules, secondary metabolites, hormones, neurotransmitters, RNAs, proteins, etc. that are in correlation with observed behavioral motifs.

- Determining if what happens in several life world circumstances correlates with the concentration of excreted molecule groups.

- Determining how behavior and molecule receptions are interconnected.

If we use this perspective it will immediately become clear that there are different levels of signaling processes in communicative actions which must be delimited if they are not to be confused with the whole variety of communication.

\section{Key Levels of Biocommunication in All Domains of Life}

There is a recognized abundance of signaling processes in all domains of life: bacteria, archaea, viruses, protozoa, fungi, animals, and plants. There are species-specific signaling processes and nichespecific signaling processes. For example, bacterial interactions with 
fungi in plant rhizosphere ecology use unique signaling to generate behavioral patterns than bacteria within the gut of animals. I have discussed this phenomenon with leading experts (Witzany 2010, 2011a, 2011b, 2012a, 2012b, 2013) and stress that there are certain key levels of biocommunication (see Fig. 2 and Appendix, p. 55) that are found throughout all species and domains of life:

- Sensing of abiotic circumstances such as light, gravity, temperature, water, dryness, wind, etc. Such environmental circumstances represent important indices on nutrition availability, symbiotic organisms, growth control, and developmental time clocks. They are not only sensed but monitored, and organisms of all domains store information about these indices in memory, to adapt better to repeated life situations.

- Transorganismic communication is termed sign-mediated interaction between non-related species, as is the case in most symbiotic partnerships or in prey-predator interaction motifs. Such transorganismic communication processes can become very complex, as in the rhizosphere of plants (together with fungi, insects, bacteria, nematodes) and also in the normal ecosphere, such as the human mouth with its 500-plus different bacterial communities.

- Interorganismic communication is termed sign-mediated interaction processes between members of the same species or related species. They share species-specific vocabulary which may vary according to different ecospheres through dialects in which the signaling semantics differs according to adaptational needs.

- Intraorganismic communication: every organism consists of various parts that must be coordinated appropriately to install life functions. The signaling processes between these parts is termed intraorganismic communication. This also includes communication between DNA storage media and genetic parasites whether they are persistent settlers of viral origin or are defectives (see Section 8.1) of infection events identified as mobile genetic elements, which now act as natural genetic engineers for better adaptation by the host organism. 


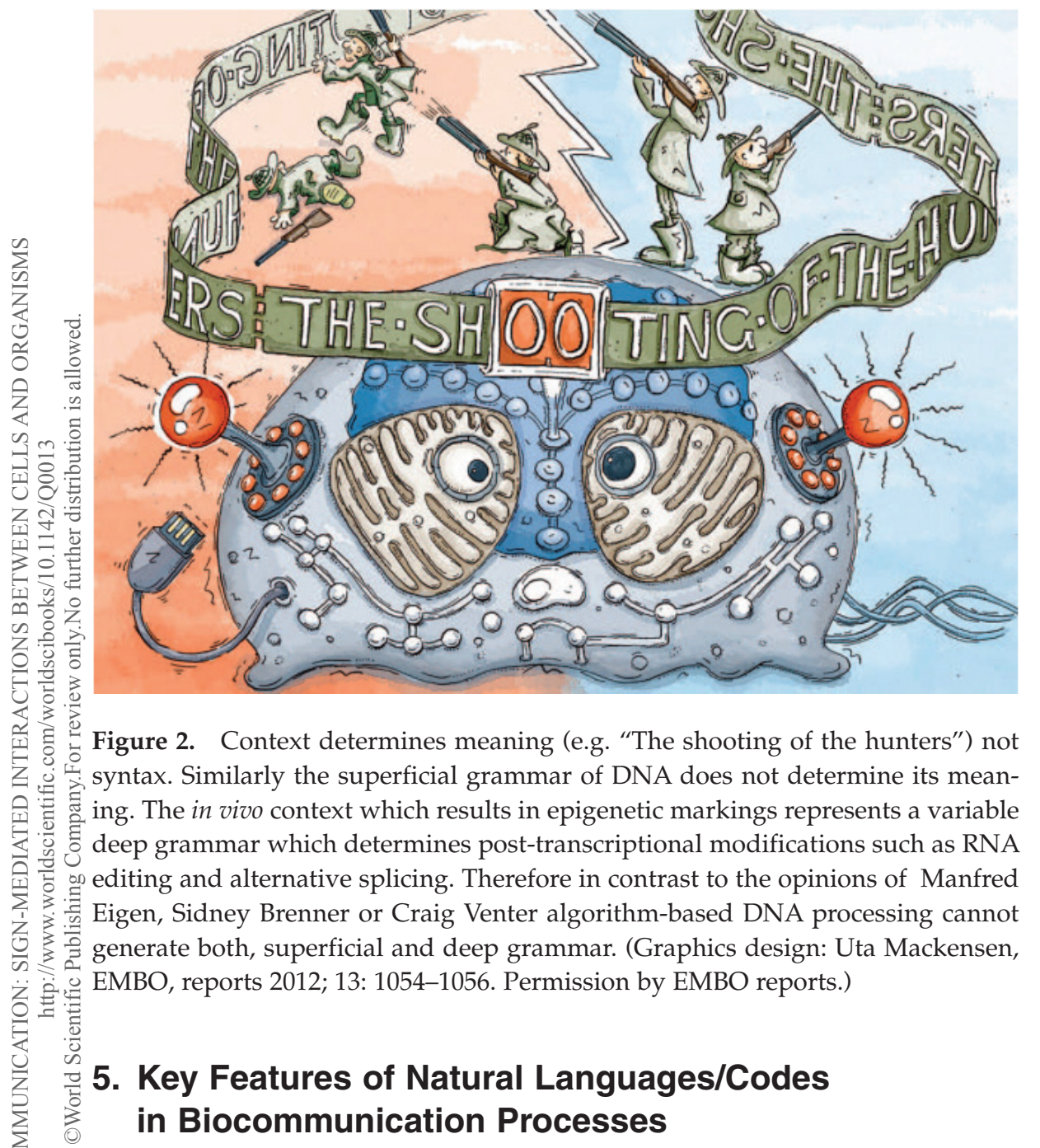

Thanks to Charles Morris we know that every natural language/ code can function within group users only if they follow three levels of rules by which the signs of a language obtain real language functions (Morris, 1946). (1) Combinatorial rules on how to correctly combine characters with larger entities such as words and sentences (syntax). (2) Context-relevant rules on how sign constructions are used according to the circumstance-dependent needs 
of the sign-using agents (pragmatics). (3) Content-dependent rules on how to correctly combine signs with designated objects.

It does not make much sense to designate ice with characters that symbolize fire. The results of empirical analyses of natural languages used by groups of humans to organize and coordinate behavior for essential life functions within a real life world are as follows:

- Natural languages/codes are tools used by groups that cannot emerge out of single individuums. Language/code usage inherently depends on social groups.

- Natural languages/codes are tools in communication processes in which members of groups interact with other members via communicative actions. This means the language tool serves to do things with signs, such as words and utterances, combined with context-dependent markings such as conscious and unconscious body expressions.

- Natural languages/codes in communicative actions function according to the intentions and goals of living agents if certain rules of usage are followed. This implies that the agents are competent in following these rules. The competence is learned and memorized within a socialization process in which rule-following in sign-use and combination to meaning is trained and later on passed from experienced to non-experienced agents.

\section{The Benefit of the Biocommunication Approach}

The advantage of a methodical adaptation of communication and linguistic terminology is that it provides appropriate tools for differentiation at specific levels, which is otherwise difficult to describe in reductive terms by means of pure physics and chemistry (molecular biology). The result is that language-like structures and communication processes occur at the simplest levels of living nature. Language and communication are not the evolutionary inventions of humans, nor are they anthropomorphic adaptations for describing non-human entities. It is an empirical fact that all coordination and organization within and between cells, tissues, organs, and organisms needs signs, i.e., molecules that serve as 
signals or symbols in messages or as vital indicators of environmental conditions. Because no natural code can encode itself, in the same way that no natural language can speak itself, these signs must be sensed and interpreted in the correct way by biological agents, i.e., there must be subjects of sign production and sign interpretation. The consequence of this is that sensing, as well as interpretation, may fail. This can result in inappropriate behavior by, or even fatal consequences for, cells, tissues, organs, and organisms.

\subsection{The machine metaphor for living organisms} is old-fashioned

The method of analyzing any part of a machine in detail to gain a picture of the whole functional blueprint, which can then be used to reproduce or manipulate it, or to produce an even more perfect example, e.g., as in artificial genetic engineering or synthetic biology, is still useful if we are dealing with machines. In contrast, communication between cells, cellular components, tissues, organs, and organisms is far from being a procedure that can be reduced to mechanistic input/output or cause/reaction descriptions (Shapiro, 2011). It is evident that communication processes between living organisms include a variety of non-mechanistic circumstances and competences that must be fulfilled in parallel if communicative acts are to have successful consequences: for example, innovating common coordination to adapt to new environmental conditions.

Machines cannot create new programs out of a functional blueprint, which is in contrast to the abilities of living organisms that are able to communicate with each other. The universal Turing machine and the self-reproducing machines of von Neumann (Turing, 1950; von Neumann, 1966; Brenner, 2012) still remain at the conceptual stage. No single self-reproducing machine has ever been identified within the last 80 years, however. There is good reason for this: it is, in principle, impossible for an artificial machine to reproduce itself (Witzany and Baluška, 2012b). In contrast to artificial machines, which cannot reproduce themselves, living cells and 
organisms can reproduce and, additionally, generate an abundance of behavioral motifs for which no algorithm can be constructed, such as de novo generation of coherent nucleotide sequences.

\section{Biocommunication and Language in Living Nature}

Consistent with current knowledge about natural communication processes, communication is defined as the sign-mediated interaction between at least two living agents which share a repertoire of signs (a kind of natural language) that are combined (according to syntactic rules) in varying contexts (according to pragmatic rules) with transport content (according to semantic rules). This means monological concepts such as the sender-receiver narrative cannot explain the emergence of commonly shared meanings.

Contrary to former concepts, the importance of this result is that these three levels of semiotic rules $(\mathrm{Gk}$ semeion $=$ sign) are complementary parts of any natural language or code. If one level is missing, according to Charles Morris, we cannot seriously speak of language or signal-mediated communication. To the best of my knowledge, the most recent definition of communication is this: sign-mediated and rule-governed interactions, i.e., interactions that depend on a shared repertoire of signs and rules (Witzany, 2010). These features are lacking in abiotic interactions, however; no semiotic rules are necessary if water freezes to ice.

\section{The Alternative to the Gene Concept: DNA as Habitat and its RNA Inhabitants}

DNA serves as a stable information storage medium and every protein which is needed by the cell is produced from this blueprint. It was recently found that an abundance of various RNA elements cooperate in a variety of steps as regulatory units with multiple competences to act on RNA transcripts. On the one hand, natural genome editing is the competent agent-driven generation and integration of meaningful DNA nucleotide sequences into pre-existing genomic content arrangements, and the ability to (re-)combine and (re-) 
regulate them according to the context-dependent (i.e., adaptational) purposes of the host organism (Witzany, 2010). Natural genome editing, on the other hand, designates the integration of all RNA activities acting on RNA transcripts without altering DNA-encoded contents. If we take the genetic code seriously as a natural code, there must be agents that are competent to act on this code because no natural code codes itself, just as no natural language speaks itself. As viral and subviral agents have been suggested as code editing agents, because there are several indicators that demonstrate viruses competent at both RNA and DNA natural genome editing (Witzany, 2013).

\subsection{Cellular genomes: stable DNA habitats for unstable RNA colonizers}

Cellular life is the main subject of biology and the history of evolution starts with the emergence of the first living cell. For decades, there was no doubt that speaking about life meant speaking about living cells and cellular assemblies. Virology elucidated the fact that viruses code for typical features that are not part of cellular life and are not found in any cell, which suggests viruses are older than cellular life (Villarreal, 2005; Forterre, 2005; Koonin, 2009). Additionally there are some indicators that viruses and viral-derived elements are the agents that edit the genome in host organisms, exemplified by the non-lytic but persistent colonization of prokaryotes and eukaryotes by viral agents (Villarreal, 2005; Villarreal and Witzany, 2010).

This view is supported by the hypothesis of a pre-cellular RNA world with an abundance of competing and cooperating ribozymes. Research on the agents that act on transcripts from the DNA information storage medium demonstrated an RNA world of high diversity and an abundance of regulatory functions in all key steps of cellular replication such as expression, transcription, translation, and repair (Gesteland et al., 2006; Witzany, 2009a, 2009b).

At the present stage we can identify two complementary kinds of natural genome editing: (1) long-lasting and stable inherited alterations of the cellular DNA genomes by persistent viral infections that alter genetic host identity and (2) co-opted adaptations of 
ribozyme-like parts as remnants of former viral colonizers that constitute regulatory elements in the present highly dynamic RNA world shortly after transcription. There are several indicators that both kinds of natural genome editing agents are epigenetically regulated (Zuckerkandl and Cavalli, 2007; Maksakova et al., 2008).

Persistent viral infection events determine gene word order in both prokaryotes and eukaryotes. Viral colonizers therefore play major roles in evolution and diversity of organisms (Villarreal, 2012). Various examples demonstrate that these viral colonizers are still active in specific developmental processes in that they are expressed in a rather limited developmental window until the process is finished. Then they are silenced again. Also well documented is the exapted function of a great variety of former retroviral parts such as env, gag, pol, that now play important roles in the gene regulation of host organisms. These "defectives" (Villarreal 2005) that now function as "effective" regulatory elements share similar features with their viral relatives and in most cases act as ribozymatic functions. They do not alter inheritable DNA content but dynamically act on RNA transcripts, although there are some examples in which RNA genome editing may also become a conserved status, especially by being reverse-transcribed into DNA.

\subsection{Agents that edit host genomes}

If the genetic code is really a natural code we must look at the agents that are able to generate sequences in this code and implement them in basic life processes such as sequence generation and context-relevant information storage and modification.

The essential assumptions after the 1960s were that natural laws determine statistical fluctuations ("genetic drift") in the molecular sequence order and replication errors ("mutations") lead to variations in DNA sequences that are the object of selection processes. The increasing volume of empirical data does not fit this concept, however:

- The central dogma of molecular biology "DNA-RNA-Protein everything else," is obsolete nowadays; the reverse trend is widespread. 
- Viruses and their defectives play various roles in evolution and developmental processes as obligate inhabitants of cellular genomes.

- Non-coding RNAs (functional RNAs that are not translated into proteins) are actively engaged in nearly all cell processes to meet both evolutionary and developmental needs.

- Mobile genetic elements (the type of DNA or RNA that can move around within a genome) that actively influence the shape of genomes and their internal interactions.

- Flexible and dynamic expansion of information processes through epigenetic programing and reprograming, alternative splicing (a regulated process occurring during gene expression that results in a single gene coding for multiple proteins), and RNA editing (a process in which changes to specific nucleotide sequences are made).

Today we can identify several key players that coordinate and organize the genetic content compositions of host organisms. They include endogenous viruses and defectives, transposons, retrotransposons, long terminal repeats, non-long terminal repeats, long interspersed nuclear elements, short interspersed nuclear elements, group I introns, group II introns, phages, and plasmids (Weiner, 2006; Slotkin and Martienssen, 2007; Kapitonov and Kohany, 2007; Lambowitz and Zimmerly, 2011). These are just some examples that use genomic DNA as their preferred living habitat. This means that DNA is not a solely a stable genetic storage medium that serves as an evolutionary protocol but is also a species-specific ecological niche for active RNAs.

A great variety of such genetic elements have been identified during the last 40 years as obligate inhabitants of all genomes, whether prokaryotic or eukaryotic. They infect, insert, and delete; some cut and paste, others copy and paste, and both spread within the genome. They change host genetic identities by insertion, recombination, or epigenetic regulation or re-regulation of genetic content, and co-evolve with the host to interact in a modular manner (Shapiro, 2011). 
Together with non-coding RNAs they shape both genome architecture and regulation. In this respect, they are agents of change not only over evolutionary time but also in real time as domesticated agents.

Non-coding RNAs interact with DNA, RNA, and proteins and play important roles in nuclear organization, transcription, posttranscriptional, and epigenetic processes (Mercer and Mattick, 2013a, 2013b). Non-coding RNAs are transcribed in both the sense and antisense directions and may be expressed in a cell type, subcellular compartment, developmental stage, or an environmental stimuli-specific, that is, context-dependent, manner. Specific RNA polymerases overlap in transcriptional contents, which means that each nucleotide can participate in varying transcriptional content arrangements according to varying contexts (Mattick, 2009, 2010).

Non-coding RNAs can be regulated in a varying manner, coordinated or independently, autonomously or functionally interrelated, and can regulate individual genes as well as large genetic networks; they can precisely control the spatiotemporal deployment of genes that are executing neuronal processes with extreme cell specificity. Various classes of non-coding RNAs target each other for post-transcriptional regulation via alternative splicing, polyadenylation, 5' capping, non-templated modifications, and RNA editing. RNA editing especially can transmit environmental information to the epigenome and therefore enable neuronal plasticity with learning and memory (Qureshi and Mehler, 2012). Additionally, non-coding RNAs can undergo nuclear-cytoplasmic, nuclear-mitochondrial, and axodendritic trafficking via ribonucleoprotein complexes that promote the spatiotemporal distribution and function of various combinations of ncRNAs, mRNAs, and RNA-binding proteins (Mercer and Mattick, 2013a, 2013b; Clark et al., 2013).

\subsection{RNA group membership: RNA stem-loop self-ligation and pseudoknots}

If we are to look at the interactional motives of RNA agents to form consortial biotic structures that follow biological selection processes 
and not mere physical-chemical reaction patterns we must look at the group building of RNA stem-loop structures (Flores et al., 2012; Witzany, 2016).

Recently it has been found that single stem-loops interact in a purely physical-chemical mode without selective forces, independently of whether they are derived randomly or are constructed under in vitro conditions (Smit et al., 2006). In contrast, if these single RNA stem-loops build groups they transcend the purely physical-chemical interaction pattern and emerge as biological selection forces, biological identities of self/non-self identification and preclusion, immune functions, dynamically changing (adapting) their membership roles. A single alteration in a base-pairing RNA stem that leads to a new bulge may dynamically alter not just this single stem-loop the whole group identity of which this stem-loop is part (Villarreal and Witzany, 2013a, 2013b).

Simple self-ligating RNA stem-loops can build much larger groups of RNA stem-loops that serve to increase complexity (Briones et al., 2009; Gwiazda et al., 2012). This may lead to ribozymatic consortia, which later on build success stories such as (1) the merger of the two subunits of transfer RNAs or (2) RNA-dependent RNA polymerases for replication of RNA through RNA or (3) the subunits of ribosomal RNAs, all of them groups that evolved and functioned for different reasons than in the later conserved modes (Dick et al., 1995; Sun and Caetano-Anolles, 2008).

Significantly, mixtures of RNA fragments that self-ligate into self-replicating ribozymes spontaneously form cooperative networks. For example, three-member networks show highly cooperative growth dynamics. When such cooperative networks compete directly against selfish autocatalytic cycles, the former grow faster, indicating the ability of RNA populations to evolve greater complexity through cooperation (Vaidya et al., 2012). In this respect cooperation clearly outcompetes selfishness.

Another intriguing example of the biological (selective) groupbuilding competence of RNA stem-loop consortia is the chemical interaction based on the molecular syntax in stem-loop "kissing," in that single-stranded regions of RNA stem-loops bind according 
to Chargaff rules to other single-stranded stem-loop structures (Staple and Butcher, 2005). It represents an RNA folding motif that links two elements of RNA secondary structure and additionally serves as a unique protein recognition site. RNA kissing motifs unite und build more complex group identities for several functions, such as dimerization of genomic RNA in viruses.

Complex three-dimensional structures can be built by consortia of single RNA sequence strings. One of the most interesting structures are pseudoknots which are composed of two helical segments connected by single-stranded regions of loops (Gupta et al., 2012). Bases in the single-stranded loop are base-paired with bases outside the loop (Staple and Butcher, 2005; Rivas and Eddy, 1999). This interaction pattern clearly depends on the rules of molecular syntax but is initiated by adaptational purposes of different ecosphere habitat dynamics. Therefore, the results of these interactions may lead to structurally diverse groups with important different biological roles such as the catalytic core of key players of the present RNA world, i.e., ribozymes (rRNAs, tRNAs), self-splicing introns, telomerase and context-dependent altered gene expression by inducing ribosomal frameshifting in several viruses.

Most interestingly, the base-pairing in pseudoknots tends to be strictly context-sensitive and base-pairing overlaps in sequence positions. Additionally, the emergence of single-nucleotide bulge loops can hardly be predicted. This currently places limitations on algorithm-based prediction models such as dynamic programming or stochastic context-free grammars (Lyngso, 2004). It indicates the Language/code nature of nucleic acid language which represents the possibility of coherent de novo generation and context-dependent alterations for a diversity of different meanings (functions) relating to the same syntax structures.

\section{Conclusions}

Biocommunication can be defined as the sign-mediated interactions of groups of living agents that share syntactic, pragmatic, and semantic rules for sign use. Biocommunication research 
investigates empirically how coordinated and organized groups of living agents function by using sign-mediated interactions as basic tools. Biocommunication cannot be sufficiently explained by mechanistic and reductionist concepts, because they cannot appropriately describe the various levels of sign use, such as sign sequences with both a superficial grammar and a deep grammar.

The biocommunication and natural genome editing approach to the processual reality of living agents has some advantages over traditional scholarly convictions: (1) a clear distinction between life and non-life, (2) an empirical non-mechanistic and non-reductionistic descriptive method of biotic interactional patterns in all organismic kingdoms. Additionally, (3) any observed coordination within and between organisms can be deciphered by research which identifies signaling molecules and syntactic, pragmatic, semantic rules underlying the mode of signal use. Last but not least, (4) biological research must no longer concentrate on experimental set-ups and theoretical approaches that seek to elucidate language and communication in non-human living nature by means of mathematical (algorithm-based) modeling.

In the light of current empirical and theoretical knowledge it is clear that the multiple levels of sign-mediated interactions which we call "communication" cannot be explained or even sufficiently described by older models such as the "sender-receiver" narrative or even based on such terms as "system" or "information." Even physical or mathematical definitions of languages as quantifiable sets of signs fail to describe the key features of these phenomena. In contrast, the biocommunication concept could serve as an appropriate complementary tool for interpreting empirical data of o biological disciplines coherent with current knowledge about communication and language.

\section{Appendix: History of Biocommunications Theory}

A first draft of a theory of biocommunication was outlined in 1971 (Tembrock, 1971). Tembrock exemplified the three semiotic levels syntax, semantics, and pragmatics in great detail for several 
behavioral patterns within the kingdom of animals. He focused on the transport of information via chemical, mechanical (tactile and acoustic) and visual signs. Although his investigations were outlined in a strict empirical manner, Tembrock justified his approach according to a solipsistic model of knowledge and communication as we came to know it in the depiction theory of language. His biocommunicative approach is therefore coherent with the senderreceiver model of information theory, a derivative from mathematical theory of language.

But the inherent features of language and communication, especially the possibility of innovative semiosis or the common understanding (and interpretation) of identical meanings, is without the realm of formalisable procedures.

In contrast with this empiricist approach, at the end of the 1980s language philosopher Guenther Witzany developed a pragmatic approach of biocommunication based on the results of the philosophy of science discourse in the $20^{\text {th }}$ century (Witzany, 1993, 2000, 2007). In this new philosophy of biology he integrated the pragmatic turn in its methodological foundation as well as the complementarity of the three semiotic levels of semiotic rules. Additionally, and in contrast with theories of knowledge with a solipsistic foundation, he investigates its scientific object according to the primacy of pragmatics, i.e., the contexts in which communicativeintersubjective sign-users are interwoven in a real life world.

The main focus of biocommunicative investigations is the agents that use and interpret signs in communicative interactions. Because 'One cannot follow rules only once' (Wittgenstein, 1953), speech and communication are kinds of social behavior and therefore it is important to investigate group behavior and group identity, the pragmatic contexts in which they are actively interwoven together with their history and cultural identity. These groups share a repertoire of signs and semiotic rules, with which they coordinate every life organization that is necessary.

This biocommunicative approach investigates communicative acts within and between cells, tissues, organs, and organisms as signmediated interactions throughout all organismic kingdoms. After developing the biocommunication approach as program in 2010, 
Witzany edited several books with leading experts in their field such as biocommunication in soil microorganisms (Witzany, 2011b), biocommunication of plants (Witzany and Baluška, 2012a), biocommunication of fungi (Witzany, 2012a), biocommunication of animals (Witzany, 2013) and biocommunication of ciliates (Witzany and Nowacki, 2016), see below. The results of these books demonstrate that competent sign-using agents from all organismic kingdoms follow syntactic, pragmatic, and semantic rules in parallel. No level of rules is reducible to one another, although in all cases context determines the meaning of the signs not syntax, i.e., the primacy of pragmatics. This is a crucial difference from all similar concepts of bringing together linguistics and biology such as systems biology, synthetic biology, biolinguistics, bioinformatics, biosemiotics, not to speak about mechanistic and even non-scientific (esoteric) approaches that want to reduce biocommunication to physicalistic and/or chemical principles.

\section{References}

Austin JL. 1975. How to do Things with Words. Harvard University Press, London.

Baluška F and Witzany G. 2013. At the dawn of a new revolution in life sciences. World Journal of Biological Chemistry, 4, 13-5.

Barbieri M. 2001. The Organic Codes. The Birth of Semantic Biology. PeQuod, Ancona.

Brenner S. 2012. Life's code script. Nature, 482, 461.

Briones C, Stich M and Manrubia SC. 2009. The dawn of the RNA world: toward functional complexity through ligation of random RNA oligomers. RNA, 15, 743-749.

Chomsky N. 1964. Current Issues in Linguistic Theory. The Hague, Mouton, London.

Clark MB, Choudhary A, Smith MA, Taft RJ and Mattick JS. 2013. The dark matter rises: the expanding world of regulatory RNAs. Essays in Biochemistry, 54, 1-16.

Dick TP and Schamel WA. 1995. Molecular evolution of transfer RNA from two precursor hairpins: implications for the origin of protein synthesis. Journal of Molecular Evolution, 41, 1-9.

Eigen M and Winkler R. 1983. The Laws of the Game: How The Principles of Nature Govern Chance. University Press, Princeton. 
Eigen M. 2013. From Strange Simplicity to Complex Familiarity a Treatise on Matter, Information, Life, and Thought. Oxford University Press, Oxford.

Flores R, Serra P, Minoia S, Di Serio F and Navarro B. 2012. Viroids: From genotype to phenotype just relying on RNA sequence and structural motifs. Frontiers in Microbiology, 3, 217. doi. 10.3389/fmicb.2012.00217.

Forterre P. 2005. The two ages of the RNA world, and the transition to the DNA world: a story of viruses and cells. Biochimie, 87, 793-803.

Gesteland RF, Cech TR and Atkins JF. 2006. The RNA World. $3^{\text {rd }}$ Edition. Cold Spring Harbor Laboratory Press, New York.

Gwiazda S, Salomon K, Appel B and Müller S. 2012. RNA self ligation: From oligonucleotides to full length ribozymes. Biochimie, 94, 1457-1463.

Habermas J. 1984. The Theory of Communicative Action. Reason and the Rationalization of Society, Vol. 1. Beacon Press, Boston.

Habermas, J. 1987. The Theory of Communicative Action. Lifeworld and System: A Critique of Functionalist Reason, Vol. 2. Beacon Press, Boston.

Habermas J. 1994. Actions, speech acts, linguistically mediated interactions and the lifeworld. Philosophical Problems Today, 1, 45-74.

Ji S. 1999. The linguistics of DNA: words, sentences, grammar, phonetics and semantics. Annals of the New York Academy of Sciences, 870, 411-417.

Koonin EV. 2009. On the origin of cells and viruses: primordial virus world scenario. Annals of the New York Academy of Sciences, 1178, 47-64.

Kuhn TS. 1967. The Structure of Scientific Revolutions. University of Chicago Press, Chicago.

Lyngsø RB. 2004. Complexity of pseudoknot prediction in simple models. Paper presented at the ICALP.

Maksakova IA, Mager DL and Reiss D. 2008. Keeping active endogenous retroviral-like elements in check: The epigenetic perspective. Cell Mol Life Sci. 65, 3329-3347

Mattick JS. 2009. Deconstructing the dogma. A new view of the evolution and genetic programing of complex organisms. Annals of the New York Academy of Sciences, 1178, 29-46.

Mattick, JS. 2010. Video Q\&A: Non-coding RNAs and eukaryotic evolution - a personal view. BMC Biology, 8, 67.

McCarthy T. 1984. Translator's introduction. In The Theory of Communicative Action 1, ix. Beacon Press, Boston.

Mercer TR and Mattick JS. 2013a. Structure and function of long noncoding RNAs in epigenetic regulation. Nature Structural \& Molecular Biology, 20, 300-307. 
Mercer TR and Mattick JS. 2013b. Understanding the regulatory and transcriptional complexity of the genome through structure. Genome Research, 23, 1081-1088.

Morris C. 1946. Signs, Language, and Behavior. Prentice Hall, New York.

Neumann VJ. 1966. Theory of Self-Reproducing Automata, 122-123. Arthur WB (ed.). University of Illinois Press, Urbana and London.

Nowak Martin AN and David C. 1999. The evolution of language. Proceedings of the National Academy of Sciences of the USA, 96, 8023-8033.

Nowak Martin AN, Joshua BP and Jansen VAA. 2000. The evolution of syntactic communication. Nature, 404, 495-498.

Nowak Martin AN, Komarova NL and Niyogi P. 2001. Evolution of universal grammar. Science, 291, 114-118.

Nowak Martin AN, Komarova NL and Niyogi P. 2002. Computational and evolutionary aspects of language. Nature, 417, 611-617.

Peirce CS. 1923. How to make our ideas clear. In Chance, Love, and Logic: Philosophical Essays. Morris RC (ed.), 41-42. Harcourt, Brace, and World, New York.

Popov O, Degal DM and Trifonov EN. 1996. Linguistic complexity of protein sequences as compared to texts of human languages. Biosystems, $38,65-74$.

Qureshi IA and Mehler MF. 2012. Emerging roles of non-coding RNAs in brain evolution, development, plasticity and disease. Nature Reviews Neuroscience, 13, 528-541.

Rivas E and Eddy SR. 1999. A dynamic programing algorithm for RNA structure prediction including pseudoknots. Journal of Molecular Biology, 285, 2053-2068.

Searle J. 1976. Speech Acts. An essay in the Philosophy of Language. Cambridge University Press, Cambridge.

Searls DB. 2002. The language of genes. Nature, 420, 211-217.

Shannon CE and Weaver W. 1949. The Mathematical Theory of Communication. University of Illinois Press, Urbana.

Shapiro JA. 2011. Evolution: A View from the 21st Century. FT Press, Washington.

Smit S, Yarus M and Knight R. 2006. Natural selection is not required to explain universal compositional patterns in rRNA secondary structure categories. RNA, 12, 1-14.

Staple DW and Butcher SE. 2005. Pseudoknots: RNA structures with diverse functions. PLoS Biology, 3(6), e213. 
60 Biocommunication

Sun FJ and Caetano-Anollés G. 2008. Transfer RNA and the origins of diversified life. PLoS One, 3(7): e2799.

Tembrock G. 1971. Biokommunikation. Informationsübertragung im biologischen Bereich. Akademie-Verlag, Berlin.

Tomasello M. 2008. Origins of Human Communication. MIT Press, Cambridge, MA.

Turing A. 1950. Computing machinery and intelligence. Mind, 59, 433-460.

Vaidya N, Manapat ML, Chen IA, Xulvi-Brunet R, Hayden EJ and Lehman N. 2012. Spontaneous network formation among cooperative RNA replicators. Nature, 49, 72-77.

Venter C. 2013. Life at the Speed of Light: From the Double Helix to the Dawn of Digital Life. Viking, New York.

Villarreal LP. 2005. Viruses and the Evolution of Life. ASM Press, Washington.

Villarreal LP. 2012. The addiction module as social force. In Viruses: Essential Agents of Life, 107-145, Springer, Dordrecht.

Villarreal LP and Witzany G. 2010. Viruses are essential agents within the roots and stem of the tree of life. Journal of Theoretical Biology, 262, 698-710.

Villarreal LP and Witzany G. 2013a. The DNA Habitat and its RNA Inhabitants. At the dawn of RNA sociology. Genomics Insights, 6, 1-12.

Villarreal LP and Witzany G. 2013b. "Rethinking quasispecies theory: From fittest type to cooperative consortia." World Journal of Biological Chemistry, 4, 79-90.

Weiner AM. 2006. SINEs and LINEs: troublemakers, saboteurs, benefactors, ancestors. In The RNA World, Gesteland RF, Cech TR and Atkins JF (eds.). 507-534. $3^{\text {rd }}$ edition. Cold Spring Harbor Laboratory Press, New York.

Witzany G. 1993. Natur der Sprache - Sprache der Natur. Sprachpragmatische Philosophie der Biologie. Königshausen \& Neumann, Wuerzburg.

Witzany G. 1995. From the 'logic of the molecular syntax' to molecular pragmatism. Explanatory deficits in Manfred Eigen's concept of language and communication. Evolution and Cognition,1, 148-168.

Witzany G. 2000. Life: The Communicative Structure. Books on Demand, Norderstedt.

Witzany G. 2007. The Logos of the Bios 2. Biocommunication. Helsinki, Umweb. 
Witzany G. 2009a. Non-coding RNAs: persistent viral agents as modular tools for cellular needs. Annals of the New York Academy of Sciences, $1178,244-267$.

Witzany G, ed. 2009b. Natural Genetic Engineering and Natural Genome Editing. New York Academy of Sciences, New York.

Witzany G. 2010. Biocommunication and Natural Genome Editing. Springer, Dordrecht.

Witzany G. 2011a. The agents of natural genome editing. Journal of Molecular Cell Biology, 3, 181-189.

Witzany G, ed. 2011b. Biocommunication in Soil Microorganisms. Springer, Heidelberg.

Witzany G, ed. 2012a. Biocommunication of Fungi. Springer, Dordrecht.

Witzany G, ed. 2012b. Viruses: Essential Agents of Life. Springer, Dordrecht.

Witzany G, ed. 2013. Biocommunication of Animals. Springer, Dordrecht.

Witzany G and Baluška F. 2012a. Biocommunication of Plants. Springer, Berlin, Heidelberg.

Witzany G and Baluška F. 2012b. "Life's code script does not code itself. The machine metaphor for living organisms is outdated." EMBO Reports, 13, 1054-1056.

Witzany G. 2016. Crucial steps to life: From chemical reactions to code using agents. Biosystems, 140, 49-57.

Witzany G and Nowacki M, eds. 2016. Biocommunication of Ciliates. Springer, Dordrecht.

Wittgenstein L. 1953. Philosophical Investigations. Basil Blackwell, Oxford, UK.

Zuckerkandl E and Cavalli G. 2007. Combinatorial epigenetics, 'junk DNA', and the evolution of complex organisms. Gene, 390, 232-242. 


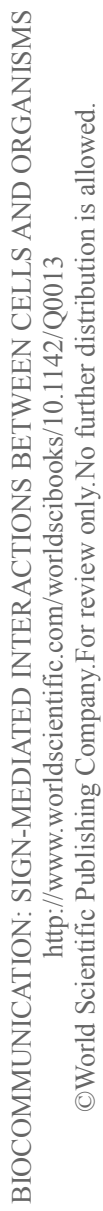

This page intentionally left blank 